\title{
Risk factors for cardiovascular events and bleeding complications following non-cardiac surgery or procedure in patients with drug eluting stent placement
}

\author{
Divya Tiwari, ${ }^{1}$ Claudine T Jurkovitz, ${ }^{2}$ Zugui Zhang, ${ }^{2}$ James Bowen, ${ }^{2}$ Paul Kolm, ${ }^{2}$ \\ Gail Wygant, ${ }^{3}$ William S Weintraub ${ }^{2}$
}

${ }^{1}$ The Royal Bournemouth and Christchurch Hospitals Foundation Trusts, Bournemouth, UK ${ }^{2}$ Christiana Care Center for Outcomes Research, Christiana Care Health System, Newark, Delaware, USA

${ }^{3}$ AstraZeneca Pharmaceuticals LP, Wilmington, Delaware, USA

Correspondence to Dr Divya Tiwari, The Royal Bournemouth and Christchurch Hospitals Foundation Trusts, Castle Lane East, Bournemouth BH7 7DW, UK ;

dtiwari@doctors.org.uk

Received 29 October 2013 Revised 24 March 2014 Accepted 29 April 2014
CrossMark

\begin{tabular}{l}
\hline To cite: Tiwari D, \\
Jurkovitz CT, Zhang Z, et al. \\
Heart Asia 2014;6:69-75. \\
doi:10.1136/heartasia-2013- \\
010471
\end{tabular}

\begin{abstract}
Objectives Previous studies suggest an increased incidence of cardiovascular (CV) events after P2Y12 receptor blocker cessation. The aim of this study was to examine the effect of $\mathrm{P} 2 \mathrm{Y} 12$ receptor blocker cessation and other risk factors on the risk of CV events and bleeding events after non-cardiac surgery/procedure in patients with drug-eluting stents (DES).
\end{abstract}

Design Retrospective cohort study.

Setting Single large healthcare system in the northeast of the USA.

Patients All adult patients who had a coronary drug eluting stent (DES) placed between 2002 and 2007 in our institution.

Interventions No randomised intervention. The principal exposure was cessation of $\mathrm{P} 2 \mathrm{Y} 12$ receptor blocker Methods This was a retrospective study of all adult patients who had a coronary DES placed between 2002 and 2007 in our institution. We considered all non-cardiac procedures up to 1 year after DES placement. Generalised estimating equations were used to identify the independent risk factors. Multiple imputations were used to replace missing values.

Main outcome measures The outcomes were CV events including death from any cause and bleeding, occurring within 30 days after the procedure.

Results From 2002 to 2007, 6397 patients had DES, $873(13.6 \%)$ had at least one non-cardiac procedure. A total of $3.6 \%$ (33/927) of the admissions were complicated by at least one cardiovascular event and 6.9\% (55/795) were complicated by bleeding. Urgent procedure (versus elective) was the only independent risk factor for CV events $(\mathrm{OR}=4.82,95 \% \mathrm{Cl} 1.95$ to 11.89$)$. Older age, diabetes, urgent procedures, orthopaedic and vascular surgery compared to unclassified surgery were independent risk factors for bleeding.

Conclusions Non-cardiac procedures are common within 1 year after DES placement. Urgent nature of procedure is a risk factor for CV events and bleeding complications. Older age, diabetes, type of surgery, are risk factors associated only with bleeding events.

\section{INTRODUCTION}

Drug-eluting coronary stents are increasingly being used to prevent the post-percutaneous coronary intervention (PCI) complication of restenosis. A large body of evidence has shown that sirolimus and paclitaxel-coated stents were highly effective in preventing restenosis. ${ }^{1}{ }^{2}$ However, sirolimus and paclitaxel may inhibit re-endothelisation of the traumatised vessel, making it vulnerable to plateletmediated thrombosis. P2Y12 receptor blockers have been instrumental in reducing the incidence of early major adverse cardiac events, including acute and late stent thrombosis after PCI. ${ }^{3} 4$

The AHA/ACC/SCAI/ACS/ADA Science Advisory Panel Guidelines recommends the use of dual antiplatelet therapy with aspirin and a P2Y12 receptor blocker for 12 months following drug-eluting stent (DES) implantation, if there is not a high risk for bleeding. ${ }^{4}$ Premature discontinuation of medication for early surgical procedures after PCI has resulted in stent thrombosis causing myocardial infarction (MI) and/or death, that has been described in the literature..$^{5-7}$ Therefore, some physicians advocate using heparin or low molecular weight heparin or glycoprotein IIb/IIIa inhibitors for 'bridge' therapy. ${ }^{89}$ On the other hand, continuing antiplatelet treatment may lead to excessive bleeding in perioperative period.

There are no clinical trial data available regarding the optimum management of patients with DES during non-cardiac surgery or other invasive procedure. Current recommendations based on guidelines and expert opinions state that if patients with DES taking a P2Y12 receptor blocker are to undergo invasive or surgical procedures, aspirin should be continued, if at all possible, and the P2Y12 receptor blocker restarted as soon as possible to avoid late stent thrombosis. Guidelines also suggest considering bare-metal stent or balloon angioplasty in patients who are likely to require invasive or surgical procedures within the 12 months following PCI. Additionally, elective procedures should be deferred for 12 months. ${ }^{8}$ However, no current guidelines exist as to the optimum management and risk of cardiovascular (CV) events in the subgroup of patients undergoing urgent surgery/invasive procedure following DES placement.

The objective of this study was to identify the risk factors for two major concerns in patients with DES who undergo non-cardiac surgery or invasive procedures. First, we aimed at identifying the risk factors associated with cardiovascular events, and second, the risk factors for intraoperative or postoperative bleeding, which at present remain an interdisciplinary challenge.

\section{METHODS}

We identified all adult patients (age $\geq 18$ years) admitted within the Christiana Care Health System 
between January 2002 and December 2007 who had a coronary DES placement. Christiana Care Health System is one of the region's largest not-for-profit healthcare providers, serving the people of Delaware as well as neighbouring Maryland, Pennsylvania and New Jersey (USA).

\section{Data sources}

Data used in this study were drawn from electronic data systems supported by Information Services at Christiana Care. Information regarding cardiac stents was extracted from the local database, which includes data collected using the American College of Cardiology National Cardiac Disease Registry-compliant software. All records were deidentified prior to analysis and the study approved by our Institutional Review Board.

\section{Variable definition}

A qualifying surgery was defined as any non-cardiac surgery/ invasive procedures occurring up to 1 year after DES placement. $\mathrm{CV}$ events were defined as a composite outcome of stroke, MI and death from any cause, happening within 30 days of surgery and identified using primary and secondary ICD9 codes, and from the discharge disposition for death (table 1).

A bleeding event was defined as any bleeding occurring 30 days after the surgery or procedure. Bleeding events were identified using primary and secondary ICD9 codes and further validated through chart review. As described in the STEEPLE study (Safety and Efficacy of Enoxaparin in Percutaneous Coronary Intervention Patients study), major bleeding was defined as any bleeding requiring a transfusion, or a surgical or endoscopic procedure ${ }^{10}$; minor bleeding was defined as any bleed that did not meet the above criteria. ${ }^{10}$

Acute MI at DES placement, diabetes and congestive heart failure (CHF) were identified using ICD9 codes (table 1). Glomerular filtration rate (GFR) was calculated using the 5variables Modification of Diet in Renal Disease equation. ${ }^{11} 12$ Presurgery serum creatinines were used to calculate GFRs. Only the measurements taken at the closest date prior to the surgery date were kept in the analysis. Chronic kidney disease (CKD) was defined as a GFR $<60 \mathrm{~mL} / \mathrm{min} / 1.73 \mathrm{~m}^{2}$.

\section{Data extraction and validation strategy}

A primary dataset was constructed by electronic extraction of information from electronic health records. Patients' records were extensively reviewed to validate and better define bleeding, to validate $\mathrm{CV}$ events, and determine the nature of surgery (elective, non-elective). Results from the electronic data extraction and from the chart review were integrated and compared. Any discrepancy in outcomes identification was further examined and reconciled. Only the hospital visits for which clopidogrel was recorded, were included in the analytical dataset.

Table 1 ICD9 codes used to identify comorbidities and outcomes

\begin{tabular}{ll}
\hline Cardiovascular events and comorbidities & ICD9-CM codes \\
\hline Stroke & $430 . x, 431 . x, 433 . x-437 . x$ \\
Acute myocardial infarction & $410 . x$ \\
Diabetes & $250 . x$ \\
Congestive heart failure & $428 . x$ \\
\hline
\end{tabular}

Statistical methods

$\chi^{2}$ Or Wilcoxon rank-sum tests were used to compare demographic and clinical characteristics according to the presence or absence of outcomes (bleeding or CV events). Observations were defined as hospital visits. Because the observations were not independent (patients may have had several hospital stays for separate occurrences of surgery), we used the technique of generalised estimating equations to identify the risk factors for $\mathrm{CV}$ events and bleeding. The generalised estimating equations allow for specification of a working covariance structure to approximate the true covariance structure of repeated $\mathrm{CV}$ events and multiple bleedings. The independent variables were age, race, gender, urgent status and timing of procedure after DES placement, whether clopidogrel was stopped, procedure type (endoscopy, orthopaedic, vascular, thoracoabdominal vs other), aspirin exposure, diabetes, heart failure (HF), CKD.

A multiple imputation procedure was used to replace each missing value among the independent variables with a set of plausible values representing the uncertainty related to the true value to impute. We also examined the interaction between urgent/elective status and the variable 'stopping clopidogrel' in the imputed models.

Data were analysed using the Statistical Analysis System V.9.1 (SAS Institute, Cary, North Carolina, USA).

\section{RESULTS}

From 2002 to 2007, 6397 patients underwent a DES placement. Of those, $873(13.6 \%)$ had one or more visits involving a noncardiac surgery or procedure within 1 year of DES placement for a total of 1219 hospital visits. Clopidogrel use was recorded in 927 visits (76.0\%). Thus, the final dataset for the cardiovascular event analyses included 927 visits for 668 patients, whereas, the final dataset for the bleeding analyses included 795 visits for 563 patients after excluding 132 visits for which bleeding preceded the index procedure/surgery and was the major cause of procedure/surgical intervention.

\section{Analyses related to the $\mathrm{CV}$ events outcome}

A total of 160 patients were treated for acute MI at initial DES placement (24.0\% of the 668 patients). Of those, 44 (27.5\%) had ST segment elevation MI. Mean age at the time of first surgery was 68.0 years (median 68.7), $75.7 \%$ were 60 years old or older, $60.2 \%$ of the patients were male and $85.3 \%$ were white. Diabetes was present among $45.5 \%$ of the patients, CHF was recorded in $34.0 \%$ and CKD in $41.7 \%$. GFR could not be calculated because of missing serum creatinine, in only $1.5 \%$ of the patients $(n=10)$.

A total of $36 \mathrm{CV}$ events (16 acute MI, 5 strokes, and 15 deaths) were recorded for 33 patients. The average time between surgery and $\mathrm{CV}$ event was 2.91 days (median $=0$, range: 0-90\%: 0-11 days).

The demographic characteristics at time of surgery between those who had and those who did not have a CV event were not statistically different. However, the patients with at least one CV event seemed to be older than those not experiencing any $\mathrm{CV}$ events, $(72.7 \%$ were $\geq 65$ years old vs $60.0 \%, p=0.1446)$, and seemed to have more comorbidities, such as diabetes $(57.6 \%$ vs $44.9 \%, \mathrm{p}=0.1534)$, CHF $(45.5 \%$ vs $33.4 \%, \mathrm{p}=0.1535)$ and CKD $(51.5 \%$ vs $41.1 \%, \mathrm{p}=0.2834)$.

As shown in table 2, $33(3.6 \%)$ of 927 surgical/invasive procedures, were complicated by at least one CV event. Of those, $63.6 \%$ were urgent (non-elective) procedures compared to $31.1 \%$ of the procedures without CV events $(\mathrm{p}<0.0001)$. The 
Table 2 Procedures and characteristics according to the presence of cardiovascular events (first event for each visit) including death from any cause among the patients who have clopidogrel exposure*

\begin{tabular}{|c|c|c|c|c|}
\hline Characteristics & $\begin{array}{l}\text { Overall } \\
n=927\end{array}$ & $\begin{array}{l}\text { Cardiovascular events } \\
\mathrm{n}=33(3.6 \%)\end{array}$ & $\begin{array}{l}\text { No cardiovascular events } \\
\mathrm{n}=894(96.4 \%)\end{array}$ & p Value \\
\hline Non-elective surgery or procedures (urgent) $(\%)$ & 32.3 & 63.6 & 31.1 & $<0.0001$ \\
\hline Average time (median) between stent placement and surgery (days) & $169.9(161.0)$ & $174.7(175.0)$ & $169.7(161.0)$ & 0.8257 \\
\hline \multicolumn{5}{|l|}{ Stopped clopidogrel } \\
\hline Yes $(\%)$ & 41.2 & 42.4 & 41.2 & \multirow[t]{3}{*}{0.7495} \\
\hline No $(\%)$ & 31.8 & 36.4 & 31.7 & \\
\hline Unknown $(\%)$ & 27.0 & 21.2 & 27.2 & \\
\hline $\begin{array}{l}\text { Among those who stopped clopidogrel prior to surgery, average (median) } \\
\text { number of days clopidogrel was stopped }\end{array}$ & $\begin{aligned} & 7.07(6.42) \\
& n=255\end{aligned}$ & $\begin{array}{l}16.20(8.53) \\
n=7\end{array}$ & $\begin{aligned} 6.81 \\
\mathrm{n}=248\end{aligned}$ & 0.0080 \\
\hline$\%$ on aspirin (documented) & 71.0 & 81.8 & 70.6 & 0.2398 \\
\hline \multicolumn{5}{|l|}{ Type of surgery } \\
\hline$\%$ endoscopy & 37.7 & 27.3 & 38.0 & \multirow[t]{5}{*}{0.0024} \\
\hline$\%$ Orthopaedic & 15.0 & 9.1 & 15.2 & \\
\hline$\%$ Vascular & 15.3 & 12.1 & 15.4 & \\
\hline$\%$ Thoracoabdominal & 11.0 & 36.4 & 10.1 & \\
\hline$\%$ Other & 21.0 & 15.1 & 21.3 & \\
\hline Any bleeding postsurgery (\%) & 5.9 & 18.2 & 5.5 & 0.0105 \\
\hline
\end{tabular}

number of days between the date of stent placement and the date of procedure was not different between those with $\mathrm{CV}$ events (median 175.0 days, IQR: 71-291 days) and those without (median 161 days, IQR: 81-251 days), $\mathrm{p}=0.8257$. Likewise, we did not find any difference in the time from stent placement to procedure between patients who had events and those who did not, whether the procedure was urgent (mean difference is 0.42 days, CI $(-48.00$ to 47.16$), \mathrm{p}=0.89)$ or elective (mean difference is 45.21 days, CI -12.06 to 102.5 ), $\mathrm{p}=0.66$ ). Stopping clopidogrel prior to surgery was not a significant risk factor for $\mathrm{CV}$ events, although it is important to note that a large proportion of these data was missing (27.0\%). The length of time between stopping clopidogrel and surgery was known in $255(66.8 \%)$ of those who stopped clopidogrel $(n=382)$. Clopidogrel was stopped for an average duration of 16.2 days (median 8.5, IQR: 7.3-14.6 days) in the group who had a CV event in postoperative period versus 6.81 days (median 6.4, IQR: $4.5-7.6$ days), $\mathrm{p}=0.0080$ in the group without CV events. Procedures with CV events were also associated with a high frequency of bleeding compared to those without any CV event $(18.2 \%$ vs $5.5 \%, \mathrm{p}=0.0105)$. A total of six patients out of $668(0.89 \%)$ experienced a CV event and bleeding postsurgery. Of the 927 surgeries/procedures, 299 were qualified as urgent. Of the urgent interventions, the most frequent were endoscopic procedures $(n=188,62.9 \%)$, followed by orthopaedic surgeries $(n=31,10.4 \%)$, thoracoabdominal surgeries $(n=29,9.7 \%)$, vascular surgeries $(n=26,8.7 \%)$ and other $(n=25,8.4 \%)$.

The only significant independent risk factor for $\mathrm{CV}$ events was the nature of the procedure (table 3). Urgent procedures were almost five times as likely to be associated with a CV event as elective procedures (table 3 ). As shown in table 3 , imputing the missing data did not modify the results. The interaction between urgent/elective status and the variable 'stopping clopidogrel' was not significant.

\section{Analyses related to the bleeding outcomes}

After excluding 132 visits for which bleeding preceded the index procedure/surgery, the demographic characteristics of the resulting population $(n=563)$ were similar to those described previously. The mean age was 67.8 years, $84.6 \%$ were white, $60.4 \%$ male, $44.4 \%$ had diabetes, $32.5 \%$ had $\mathrm{CHF}$ and $40.0 \%$ had CKD. GFR could not be calculated because of missing serum creatinine, in only $1.8 \%$ of the patients $(n=10)$.

A total of 55 postoperative bleeding events were recorded for 52 patients; 41 bleeding events were determined to be minor, 14 met the criteria for major bleeding. The average time between surgery and bleeding events was 2.81 days (median $=0$, IQR 0-3 days). Compared to the patients who did not have a postoperative bleeding event, patients who had a bleeding event were older $(73.1 \%$ were $\geq 65$ years vs $59.3 \%, p=0.0527)$ and were more likely to have diabetes $(59.6 \%$ vs $42.9 \%$, $\mathrm{p}=0.0205)$. Race, gender, presence of CHF and CKD were not statistically different between the two groups.

As shown in table 4, out of 795 surgical/invasive procedures, $55(6.9 \%)$ were complicated by at least one bleeding event. Among the procedures complicated by a bleeding event, $43.6 \%$ were urgent (non-elective) procedures compared to only $20.7 \%$ among those without bleeding events $(p<0.0001)$.

The average duration between stent placement and surgery was 173.1 days (median=167.0, IQR: 87-254 days). The length of time between stent placement and surgery was not different between those with bleeding events and those without. Likewise, we did not find any difference in the time from stent placement to procedure between patients who had bleeding events and those who did not whether the procedure was urgent (mean difference is 1.74 days, CI (-46.49 to 43.01), $\mathrm{p}=0.51$ ) or elective (mean difference is 1.38 days, CI -34.89 to $37.65), p=0.97)$. The length of time between stopping clopidogrel and surgery was known in 248 (79.5\%) of those who stopped clopidogrel $(n=312)$. The average number of days clopidogrel was stopped prior to surgery was not different between the two groups (8.9 days (median 7.4, IQR: 5.5-9.4 days) vs 6.7 days (median 6.4, IQR: 4.5-7.6 days), $\mathrm{p}=0.1577$ ).

Dual antiplatelet therapy (aspirin plus clopidogrel) was used more frequently among the patients who experienced bleeding than among those who did not $(81.8 \%$ vs $67.7 \%, \mathrm{p}=0.0341)$.

Among the procedures complicated by a bleeding event, $49.1 \%$ were orthopaedic compared to only $15.1 \%$ among those without bleeding events $(p<0.0001)$. A total of $7(50.0 \%)$ of 
Table 3 Significant independent risk factors associated with CV events

\begin{tabular}{|c|c|c|c|c|c|c|}
\hline & \multicolumn{3}{|c|}{ Original data $(n=915)^{*}$} & \multicolumn{3}{|c|}{ Imputed data $(n=927)$} \\
\hline & $\mathrm{OR}$ & $95 \% \mathrm{Cl}$ & $\mathrm{p}$ Value & OR & $95 \% \mathrm{Cl}$ & $\mathrm{p}$ Value \\
\hline Male vs female (ref) & 0.63 & 0.27 to 1.48 & 0.2844 & 0.84 & 0.49 to 1.51 & 0.3852 \\
\hline Age $\geq 65$ vs $<65$ (ref) & 1.55 & 0.60 to 3.95 & 0.3639 & 1.65 & 0.84 to 3.27 & 0.2144 \\
\hline Non-white vs white (Ref) & 0.31 & 0.07 to 1.27 & 0.1025 & 0.50 & 0.21 to 1.18 & 0.1458 \\
\hline \multicolumn{7}{|l|}{ Type of surgery } \\
\hline Urgent vs elective (ref) & 4.99 & 2.07 to 11.96 & 0.0003 & 4.95 & 2.67 to 10.08 & $<0.0001$ \\
\hline \multicolumn{7}{|l|}{ Stopped clopidogrel } \\
\hline No (ref) & 1 & & & 1 & & \\
\hline Yes & 0.79 & 0.30 to 2.11 & 0.6384 & 0.77 & 0.39 to 1.45 & 0.4289 \\
\hline Unknown & 0.77 & 0.24 to 2.78 & 0.6551 & & & \\
\hline Aspirin & 1.33 & 0.44 to 4.08 & 0.6139 & 0.95 & 0.47 to 1.98 & 0.5347 \\
\hline Time from stent to procedure & 1.00 & 0.99 to 1.00 & 0.5868 & 1.00 & 0.99 to 1.00 & 0.6247 \\
\hline \multicolumn{7}{|l|}{ Type of surgery } \\
\hline Unclassified (ref) & 1 & 0.12 to 2.26 & 0.3760 & 1 & 0.15 to 1.05 & 0.0658 \\
\hline Endoscopy & 0.51 & 0.10 to 2.71 & 0.4432 & 0.43 & 0.16 to 1.40 & 0.1652 \\
\hline Orthopaedic & 0.53 & 0.18 to 5.16 & 0.9565 & 0.44 & 0.35 to 2.39 & 0.6745 \\
\hline Vascular & 0.95 & 0.87 to 11.72 & 0.0820 & 0.81 & 0.82 to 5.18 & 0.0875 \\
\hline Thoracoabdominal & 3.18 & & & 2.11 & & \\
\hline Diabetes & 2.04 & 0.78 to 5.30 & 0.1446 & 1.65 & 0.80 to 3.21 & 0.1705 \\
\hline CHF & 0.78 & 0.32 to 1.91 & 0.5899 & 1.20 & 0.61 to 2.32 & 0.5899 \\
\hline CKD $(G F R<60)$ vs no CKD $(G F R \geq 60)$ & 0.83 & 0.37 to 1.84 & 0.6479 & 1.04 & 0.54 to 1.91 & 0.8246 \\
\hline
\end{tabular}

* The number of records used for the GEE analysis is 915 because serum creatinine was missing for 11 records and type of surgery was missing for one record; the 250 records that were missing information about stopping clopidogrel were categorised as unknown in the non-imputed model.

CHF, congestive heart failure; CKD, chronic kidney disease; CV, cardiovascular; GEE, generalised estimating equation; GFR, glomerular filtration rate.

the 14 surgeries with major bleeding happened under continued clopidogrel; clopidogrel was discontinued for 5 (35.7\%), and clopidogrel status was unknown for $2(14.3 \%)$. The types of surgery complicated with major bleeding were orthopaedic $(n=6,42.9 \%)$, vascular $(n=5,35.7 \%)$ and endoscopy $(n=3$, 21.4\%).

A total of 177 interventions of the 795 visits were qualified as urgent. Of the urgent interventions, the most frequent was endoscopy $(n=86,48.6 \%)$, orthopaedic $(n=31,17.5 \%)$, thoracoabdominal $(n=24,13.6 \%)$, vascular $(n=22,12.4 \%)$ and other $(n=14,7.9 \%)$.

CV events were also more frequent among those who experienced bleeding than among those who did not (10.9\% vs $2.8 \%$, $\mathrm{p}=0.0079$ ).

Independent risk factors for postprocedure bleeding in patients with DES included older age and diabetes, urgent

Table 4 Procedures and characteristics according to the presence of postsurgical bleeding event (first bleeding event for each visit) among the patients who have clopidogrel exposure*

\begin{tabular}{|c|c|c|c|c|}
\hline & $\begin{array}{l}\text { Overall } \\
n=795\end{array}$ & $\begin{array}{l}\text { Bleeding event } \\
\mathrm{n}=55(6.9 \%)\end{array}$ & $\begin{array}{l}\text { No bleeding event } \\
\mathrm{n}=740(93.1 \%)\end{array}$ & p Value \\
\hline Non-elective surgery or procedures (urgent) (\%) & 22.3 & 43.6 & 20.7 & $<0.0001$ \\
\hline Average time (median) between stent placement and surgery (days) & $173.1(167.0)$ & $167.1(146.0)$ & $173.6(168.0)$ & 0.6096 \\
\hline Stopped clopidogrel & & & & 0.2806 \\
\hline Yes $(\%)$ & 39.3 & 41.8 & 39.1 & \\
\hline No (\%) & 31.7 & 38.2 & 31.2 & \\
\hline Unknown (\%) & 29.1 & 20.0 & 29.7 & \\
\hline $\begin{array}{l}\text { Among those who stopped clopidogrel, average (median) } \\
\text { number of days clopidogrel was stopped prior to surgery }\end{array}$ & $\begin{array}{ll}6.83(6.42) \\
n=248\end{array}$ & $\begin{array}{ll}8.90 \\
\mathrm{n}=15\end{array}$ & $\begin{aligned} 6.70 \\
\mathrm{n}=233\end{aligned}$ & 0.1577 \\
\hline$\%$ on aspirin & 68.7 & 81.8 & 67.7 & 0.0341 \\
\hline \multicolumn{5}{|l|}{ Type of surgery } \\
\hline \% endoscopy & 30.6 & 14.6 & 31.8 & $<0.0001$ \\
\hline$\%$ Orthopaedic & 17.5 & 49.1 & 15.1 & \\
\hline$\%$ Vascular & 17.4 & 23.6 & 16.9 & \\
\hline$\%$ Thoracoabdominal & 12.2 & 5.4 & 12.7 & \\
\hline$\%$ Other & 22.4 & 7.3 & 23.5 & \\
\hline Any CV event (\%) & 3.4 & 10.9 & 2.8 & 0.0079 \\
\hline
\end{tabular}

*The denominator in this table is surgical visits.

$\mathrm{CV}$, cardiovascular. 
nature of procedure, type of surgery, for example, orthopaedic surgery and vascular surgery compared to unclassified surgery (table 5). As shown in table 5, imputing the missing data did not modify the results.

\section{DISCUSSION}

The principal findings of this study are, first, among patients who underwent DES placement, almost $14.0 \%$ had one or more visits involving a non-cardiac surgery or procedure within 1 year of DES placement and, second, the incidence of CV events per surgery/procedure was $3.6 \%$ and the incidence of bleeding events $6.9 \%$. Urgent surgical procedures were almost five times as likely to result in a CV event and three times as likely to result in a bleeding event as elective surgery. In this analysis, the urgent characteristic of a procedure was the only independent risk factor for $\mathrm{CV}$ events. Other independent risk factors for bleeding included older age, diabetes and orthopaedic and vascular surgery compared to unclassified surgery.

Coronary stent insertion is the most preferred PCI for coronary revascularisation. ${ }^{3}$ DES are increasingly used on-label and off-label, in approximately $70-80 \%$ of all PCIs in the USA because of increased procedural success and low rate of restenosis. ${ }^{8}$ Previous studies have shown that the temporary discontinuation of antiplatelet therapy prior to non-cardiac surgery/procedures increases the risk of perioperative stent thrombosis, but continued therapy increases the risk of surgical bleeding. ${ }^{4} 13{ }^{14}$ No prospective randomised clinical trial data are available to address the problem of premature discontinuation of therapy. A randomised trial in which clopidogrel therapy is stopped would likely raise ethical issues.

Kaluza et $a l,{ }^{13}$ in a retrospective study of 40 patients who underwent coronary stent placement between 1996 and 1998, were the first to report a large perioperative mortality $(20 \%)$ following non-cardiac surgery early (less than 6 weeks) after stent insertion. Among patients who died, 75\% $(n=6)$ suffered a $\mathrm{MI}$ in the postoperative period while $25 \%$ experienced a bleeding complication $(n=2)$. Since then and with the increased use of DES, many case reports of perioperative stent thrombosis following non-cardiac surgery have been published, but few studies have reported results from large number of patients. Describing results from various small retrospective studies, with the number of patients varying from 38 to 141 , authors have reported major CV events rates ranging from $0 \%$ to $5 \%$ after DES implantation, with the average number of days between stent implantation and surgery varying between approximately 228 and 260 days $^{6} 1516$ and up to 14 months in one study. ${ }^{7}$ In a more recent retrospective study of 520 patients who underwent non-cardiac surgery after DES placement, the investigators found the rate of major adverse cardiac event (MACE) to be approximately 6\% within a year of stent placement. ${ }^{14}$ This higher rate may be explained by the fact that bleeding complications were included in the definition of MACE. On the other hand, in a prospective observational multicentre study of 103 patients who underwent a bare-metal stent or DES implantation, Vicenzi et $a l^{9}$ reported a frequency of adverse events of $44.7 \%$ and a cardiac mortality rate of $4.9 \%$ after non-cardiac surgery. It is important to note, however, that their definition of adverse events included CV events, coronary revascularisation, CHF, significant arrhythmias, troponin leaks, bleeding, surgical complications, sepsis. Finally, in a retrospective analysis of 41989 surgeries and procedures occurring within 24 months of a coronary stent implantation in 28029 Veterans Affairs (VA) patients between 2000 and 2010, Hawn et $a l^{17}$ found that $22.5 \%$ of the patients underwent surgery within 24 months and $4.7 \%$ of the surgeries resulted into a major cardiac event within 30 days. We found lower frequency of surgeries and of cardiac events $(14.0 \%$ and $3.6 \%)$ because we only examined the surgeries

Table 5 Significant independent risk factors associated with bleeding

\begin{tabular}{|c|c|c|c|c|c|c|}
\hline & \multicolumn{3}{|c|}{ Original data $\left(n=783^{*}\right)$} & \multicolumn{3}{|c|}{ Imputed data $(n=795)$} \\
\hline & OR & $95 \% \mathrm{Cl}$ & $\mathrm{p}$ Value & OR & $95 \% \mathrm{Cl}$ & $\mathrm{p}$ Value \\
\hline Male vs female (ref) & 1.75 & 0.86 to 3.58 & 0.1243 & 1.71 & 0.82 to 3.35 & 0.1458 \\
\hline Age $\geq 65$ vs <65 (ref) & 2.46 & 1.17 to 5.19 & 0.0181 & 2.50 & 1.21 to 5.08 & 0.0121 \\
\hline Non-white vs white (ref) & 0.74 & 0.26 to 2.13 & 0.5730 & 0.73 & 0.26 to 2.05 & 0.5417 \\
\hline \multicolumn{7}{|l|}{ Type of surgery } \\
\hline Urgent vs elective (ref) & 4.08 & 1.97 to 8.43 & 0.0002 & 2.87 & 1.42 to 5.15 & 0.0023 \\
\hline \multicolumn{7}{|l|}{ Stopped clopidogrel } \\
\hline No (ref) & 1 & & & 1 & & \\
\hline Yes & 1.36 & 0.61 to 3.02 & 0.4578 & 1.18 & 0.51 to 2.12 & 0.7459 \\
\hline Unknown & 0.82 & 0.34 to 1.96 & 0.6457 & & & \\
\hline Aspirin & 1.56 & 0.68 to 3.57 & 0.2905 & 1.52 & 0.71 to 3.18 & 0.2143 \\
\hline Time from stent to procedure & 1.00 & 0.99 to 1.00 & 0.5847 & 1.00 & 0.99 to 1.00 & 0.6284 \\
\hline \multicolumn{7}{|l|}{ Type of surgery } \\
\hline Unclassified (ref) & 1 & & & 1 & & \\
\hline Endoscopy & 0.91 & 0.27 to 3.08 & 0.8822 & 0.67 & 0.17 to 2.32 & 0.5204 \\
\hline Orthopaedic & 7.88 & 2.67 to 23.29 & 0.0002 & 10.15 & 3.42 to 10.11 & $<0.0001$ \\
\hline Vascular & 4.06 & 1.25 to 13.15 & 0.0110 & 4.66 & 1.25 to 14.28 & 0.0012 \\
\hline Thoracoabdominal & 0.74 & 0.16 to 3.51 & 0.7053 & 1.01 & 0.25 to 4.09 & 0.9146 \\
\hline Diabetes & 1.99 & 1.02 to 3.87 & 0.0426 & 2.01 & 1.14 to 3.85 & 0.0201 \\
\hline CHF & 0.68 & 0.31 to 1.50 & 0.3370 & 0.65 & 0.27 to 1.32 & 0.2158 \\
\hline CKD $(G F R<60)$ vs no $C K D(G F R \geq 60)$ & 0.70 & 0.31 to 1.57 & 0.3820 & 0.66 & 0.25 to 1.51 & 0.2058 \\
\hline
\end{tabular}

*The number of records used for the GEE analysis is 783 because serum creatinine was missing for 11 records and type of surgery was missing for one record. The 231 records that were missing information about stopping clopidogrel were categorised as unknown in the non-imputed model.

$\mathrm{CHF}$, congestive heart failure; CKD, chronic kidney disease; GEE, generalised estimating equation; GFR, glomerular filtration rate. 
within 1 year of stenting, and did not include revascularisation as a major cardiac event as did Hawn et al. ${ }^{17}$

Time of discontinuation of antiplatelet agents, presence of diabetes, CKD, HF, and presence of tumour have been described as risk factors for CV events. ${ }^{9}$ Likewise, some studies report time between stent placement and surgery as the main determinant of outcome. ${ }^{9} 1318$ In a recent systematic review describing the results of 28 carefully selected studies (out of 358 ), the authors concluded that there was enough evidence to support a significant decrease in major adverse cardiac events when surgery was performed more than 1 year after DES placement. ${ }^{19}$ No conclusive evidence was found to demonstrate the benefit of continuing perioperative antiplatelet therapy. ${ }^{19}$

Similar to the previous recent studies, we reported that $3.6 \%$ of surgical procedures after stent placement were complicated by at least one CV event. The only independent risk factor for CV events was the urgent nature of surgery. In our study, stopping clopidogrel or timing of the procedure in relation to the stent placement did not have an independent effect on the occurrence of CV events. However clopidogrel was stopped for a longer average duration prior to surgery in patients who experienced CV events when compared with the patients who had no CV events (16.2 vs 6.8 days $\mathrm{p}=0.008)$. Because we were concerned that the results could be biased by the large number of missing data, we ran a second model that included imputed data for all variables with missing data. The results between the two models were similar, indicating that the data were missing at random. Our results are similar to the results described by Hawn $e a^{17}$ in their study of 28029 VA patients. They found that after adjustment, non-elective surgical admission was strongly associated with major CV events (adjusted OR, 4.77; 95\% CI 4.07 to 5.59), and did not find an association between antiplatelet cessation and major CV events (OR, 0.86; 95\% CI 0.57 to 1.29). Therefore, our results complement those of Hawn et al because our population is more diverse $(60.2 \%$ male in our population vs $98.4 \%$ in the VA population of Hawn et al study; younger $(75.8 \%$ of our population is 60 years old or more vs $80.6 \%$ of the VA population) and have less comorbidities $34.0 \%$ of our population has CHF, $45.5 \%$ has diabetes vs $43.1 \%$ and $49.4 \%$, respectively, in the VA population).

Bleeding was frequent in our large study population, and was associated with older age, urgent versus elective surgery, orthopaedic and vascular surgeries and diabetes. Similar to the analysis of CV events, we did not find that stopping clopidogrel or timing of the procedure in relation to the stent placement had an effect on the occurrence of bleeding.

As others did, we also found that postoperative bleeding was more frequent among patients with CV events, and CV events were more frequent among those who bled. ${ }^{13}$ We can hypothesise that interrupting antiplatelet agents in patients who bleed may increase the risk for a CV event and keeping antiplatelet agents in patients who have had a CV event may increase bleeding. Unfortunately, we do not know whether the CV event preceded the bleeding or vice-versa. The association of $\mathrm{CV}$ event and bleeding has been reported in previous studies. In a retrospective study of 40 patients undergoing non-cardiac surgery after stent placement, Kaluza et $a l^{13}$ reported that four patients $(10.0 \%)$ had MI and bleeding. All patients were taking ticlopidine and aspirin prior to surgery, and two patients had ticlopidine and aspirin withheld prior to surgery. Likewise, in a more recent prospective study of 103 patients, Vicenzi et al ${ }^{9}$ reported that two patients (1.9\%) experienced MI and bleeding. The rate of experiencing both events is much lower in our population, probably because our patients were under a different anticoagulation regimen, and all had a DES, which was not the case for the two previous studies.

\section{LIMITATIONS}

The data used in this study were extracted from administrative databases, although the patients' charts were extensively reviewed to confirm the occurrence of events.

Whether clopidogrel was stopped or not was difficult to ascertain despite a thorough chart review. Even though the models' results were similar prior to and after data imputation, the large proportion of missing data prevents inferring any conclusion on the effect of stopping clopidogrel.

\section{CONCLUSION}

In conclusion, non-cardiac surgeries/procedures are not rare 1 year after coronary stent placement (14\%), and approximately $4 \%$ of them are complicated by a CV event while $7 \%$ are complicated by a bleeding event. More than the timing of the procedure in relation to the stent placement, the relative urgency of the surgery/procedure is a major risk factor for $\mathrm{CV}$ events and bleeding. Both risks should be carefully evaluated and balanced at the time of decision for urgent non-cardiac surgery/procedures in this patient population. None of the bleeding events were life threatening or fatal, but their effect on outcomes of surgery in terms of cost implications, because of prolonged hospital stay or need for blood transfusion, warrants further evaluation and careful consideration.

Acknowledgements The authors thank Ashley Weed for her invaluable assistance in setting up the database for analysis.

Contributors Primary researchers: DT, CTJ, WSW, GW. Biostatistician: PK, ZZ. Data set Construction: JB.

Funding This work was funded by a grant from AstraZeneca Pharmaceuticals $L P$, Wilmington, DE.

\section{Competing interests None.}

Ethics approval Study was approved by the Institutional Review Board USA.

Provenance and peer review Not commissioned; externally peer reviewed.

\section{REFERENCES}

1 Colmenarez HJ, Escaned J, Fernández C, et al. Efficacy and safety of drug-eluting stents in chronic total coronary occlusion recanalization: a systematic review and meta-analysis. J Am Coll Cardiol 2010;55:1854-66.

2 James SK, Stenestrand U, Lindbäck J, et al. Long-term safety and efficacy of drug-eluting versus bare-metal Stents in Sweden. N Eng J Med 2009;360:1933-45.

3 Metzler H, Kozek-Langenecker S, Huber K. Antiplatelet therapy and coronary stents in perioperative medicine-the two sides of the coin. Clinical Anaesthesiology 2008;22:81-94.

4 Grines $\mathrm{CL}$, Bonow RO, Casey DE, et al. Prevention of premature discontinuation of dual antiplatelet therapy in patients with coronary artery stents. Circulation 2007;115:813-18.

5 Pfisterer M, Brunner-La Rocca HP, Buser PT, et al. Late clinical events after clopidogrel discontinuation may limit the benefit of drug-eluting stents: an observational study of drug-eluting versus bare-metal stents. J Am Coll Cardiol 2006;48:2584-91.

6 Rhee SJ, Yun $\mathrm{KH}$, Lee SR, et al. Drug eluting stent thrombosis during perioperative period. Int Heart J 2008;49:135-42.

7 Godet G, Le Manach Y, Lesache F, et al. Drug-eluting stent thrombosis in patients undergoing non-cardiac surgery:is it always a problem? Br J Anaesth 2008;100:472-7.

8 Brilakis ES, Banerjee S, Berger PB. Peroperative management of patients with coronary stents. J Am Coll Cardiol 2007:49:2145-50.

9 Vicenzi MN, Meislitzer T, Heitzinger B, et al. Coronary artery stenting and noncardiac surgery- a prospective outcome study. Br J Anaesth 2006;96:686-93.

10 Montalescot G, White HD, Gallo R, et al. Enoxaparin versus unfractionated heparin in elective percutaneous coronary intervention. STEEPLE Investigators. N Engl J Med 2006;355:1006-17.

11 Levey AS, Bosch JP, Lewis JB, et al. A more accurate method to estimate glomerular filtration rate from serum creatinine: A new prediction equation. Modification of Diet in Renal Disease Study Group. Ann Intern Med 1999;130:461-70. 
12 Levey AS, Coresh J, Greene T, et al. Using standardized serum creatinine values in the Modification of Diet in Renal Disease study equation for estimating glomerular filtration rate. Ann Int Med 2006:145:247-54.

13 Kaluza GL, Joseph J, Lee JR, et al. Catastrophic outcomes of noncardiac surgery soon after coronary stenting. J Am Coll Cardiol 2000;35:1288-94.

14 Rabbitts JA, Nuttall GA, Brown MJ, et al. Cardiac risk of noncardiac surgery after percutaneous coronary intervention with drug-eluting stents. Anesthesiology 2008; 109:596-604.

15 Compton PA, Zankar AA, Adesanya AO, et al. Risk of noncardiac surgery after coronary drug-eluting stent implantation. Am I Cardiol 2006;98:1212-13.
16 Brotman DJ, Bakhru M, Saber W, et al. Discontinuation of antiplatelet therapy prior to low-risk noncardiac surgery in patients with drug-eluting stents: a retrospective cohort study. J Hosp Med 2007;2:378-84.

17 Hawn MT, Graham LA, Richman JS, et al. Risk of major adverse cardiac events following noncardiac surgery in patients with coronary stents. JAMA 2013;310:1462-72.

18 Schouten 0 , van Domburg RT, Bax JJ, et al. Noncardiac surgery after coronary stenting: early surgery and interruption of antiplatelet therapy are associated with an increase in major adverse cardiac events. J Am Coll Cardiol 2007;49:122-4.

19 Hollis RH, Graham LA, Richman JS, et al. Adverse cardiac events in pateitns with coronary stents undergoing noncardiac surgery: a systematic review. Am I Surgery 2012;204:494-501. 